\title{
Online Time Delay Identification and Control for General Classes of Nonlinear Systems
}

\author{
Alper Bayrak and Enver Tatlicioglu
}

\begin{abstract}
In this study, online identification of state delays is discussed. First, a novel adaptive time delay identification technique is proposed for general classes of autonomous nonlinear systems subject to state delays. As an extension, this technique is modified to design a tracking controller for general classes of nonlinear systems subject to state delays. The main novelty of this controller is that identification of unknown state delays is ensured while output tracking objective is satisfied. Extensive numerical simulations are presented that demonstrate the efficiency of the time delay identification algorithm and the tracking controller.
\end{abstract}

\section{INTRODUCTION}

Time delay, also named as time difference of arrival or dead time in different disciplines, is an important research area mostly due to its negative effects (such as instability or reduced performance) on systems [1]. Time delay may originate from the dynamics of systems, or may be introduced by feedback loops, sensors, and communication lines.

Since time delay is a real problem that occurs in several systems, significant amount of research has been conducted on its effects on stability, and identification and control methods for time delayed systems. A broad overview on time delay and its effects on systems may be found in Richard's work [1]. Niculescu and $\mathrm{Gu}$ also presented a broad overview, that particularly focused on engineering applications and recent progresses of stability and control of time delay systems [2].

A significant amount of research was devoted to designing time delay identification algorithms [3], [4], [5], [6], [7], [8], [9], [10], [11], [12], [13], [14]. Most of the past research on time delay identification were usually presented for linear or linearized systems and review of the relevant past research highlights the fact that there are no time delay identification algorithms for general classes of nonlinear systems.

Due to the negative effects of time delay on stability and performance, a significant amount of research was devoted to designing controllers for systems subject to time delays. Gu et al. [2] and Zhong [15] investigated robust control and robust stability of time delay systems. Schoen investigated the stability of time delay systems by using Razumikhin theory, Lyapunov-Krasovskii theory, and eigenvalue consideration [16]. In [17], Krstic focused on systems with input delays and converted the problem to boundary control of partial differential equations after introducing a transformation. Niculescu analyzed effects of time delays on stability of dynamical

\footnotetext{
The authors are with the Department of Electrical \& Electronics Engineering, Izmir Institute of Technology, Urla, Izmir, 35430 Turkey.

\{alperbayrak, envertatlicioglu\}@iyte.edu.tr
}

systems [18]. In [19], Mazenc et al. presented results of robustness with respect to delay in the input and gave proofs based on Lyapunov-Krasovskii functionals constructions.

For nonlinear systems subject to state delays, accurate knowledge of time delays is advantageous for control development, however time delay is usually unknown. To overcome this problem, estimating time delay while controlling the system may be an effective method. In [20], Peng et al. considered the Smith predictor based controller design for network control systems with time delay identification. Zhang and Li presented a fuzzy Smith predictor based controller for time-varying processes based on time delay identification for signal processing applications [21]. In [22], Zhang and $\mathrm{Li}$ proposed a control method for teleoperation systems based on time delay identification. Review of the relevant literature highlights the fact that there are no notable control approaches based on time delay identification. The approaches in the literature are usually valid for some special cases, and not for general nonlinear systems.

In this work, first, general classes of autonomous nonlinear systems subject to state delays is considered and a novel time delay identification technique is proposed. While designing the identification algorithm, the time delay is considered as a nonlinear parameter affecting the system, and the nonlinear parameter identification method in [23] is utilized as the time delay identification method. In the design of the time delay identification algorithm, auxiliary observer-like signals are designed. The stability of the closed-loop system and the convergence of the time delay identification can be proven via Lyapunov based methods. When compared with the literature, the proposed time delay identification is designed via Lyapunov based methods, it works online, and it can be applied to general classes of nonlinear systems without imposing any restrictions. As an extension, general classes of nonlinear systems subject to state delays is considered and a tracking controller is designed. The main novelty of this part is that while the controller ensures tracking of a desired trajectory, state delays can be identified online. Extensive numerical simulations are presented that demonstrate the validity of the time delay identification algorithm and the tracking controller. 


\section{SySTEM Model}

The following system is considered

$$
\begin{aligned}
& \dot{x}_{1}=x_{2} \\
& \vdots \\
& \dot{x}_{m-1}=x_{m} \\
& \dot{x}_{m}=f(x, \tau, t)
\end{aligned}
$$

where $f(\cdot) \in \mathbb{R}$ is a nonlinear function, $x(t)=$ $\left[\begin{array}{ll}x_{1} & x_{2} \cdots x_{m}\end{array}\right] \in \mathbb{R}^{m}$ is state vector, and $\tau \in \mathbb{R}^{n}$ denotes unknown constant time delay vector. In this system, derivative of $m^{\text {th }}$ component of state vector may consist of all components of the state vector and their delayed forms (for example, see (36) for the mathematical model of chattering phenomenon during metal cutting operation). It is assumed that the structure of $f$ is known and the state vector $x(t)$ is measurable. As a consequence of the properties of the differential model in (1), $x(t)$ is continuous, bounded, and Lipschitz in time as

$$
\left\|x\left(t_{1}\right)-x\left(t_{2}\right)\right\| \leq L_{1}\left|t_{1}-t_{2}\right| \quad \forall t_{1}, t_{2} \in \mathbb{R}^{+}
$$

where $L_{1} \in \mathbb{R}$ is a positive Lipschitz constant.

Assumption 1: It is assumed that $\tau$, the unknown time delay vector, is bounded and is in a known hypercube $\Omega \subset$ $\mathbb{R}^{n}$.

Assumption 2: It is assumed that, for any $x$ and $t$, the function $f$ is either concave or convex on a simplex ${ }^{1} \Omega_{s}$ in $\mathbb{R}^{n}$, and also $\Omega_{s} \supset \Omega$.

Assumption 3: It is assumed that $f\left(\tau_{0}, x, t\right)$ is Lipschitz with respect to its arguments in the sense that

$$
\begin{array}{r}
\left|f\left(\tau_{0}+\Delta \tau_{0}, x+\Delta x, t+\Delta t\right)-f\left(\tau_{0}, x, t\right)\right| \\
\leq L_{2}\left(\left\|\Delta \tau_{0}\right\|+\|\Delta x\|+\|\Delta t\|\right)
\end{array}
$$

where $\Delta x \triangleq x\left(t_{1}\right)-x\left(t_{2}\right), \Delta \tau_{0} \triangleq \tau_{0}\left(t_{1}\right)-\tau_{0}\left(t_{2}\right), \Delta t \triangleq$ $t_{1}-t_{2}$, and $L_{2} \in \mathbb{R}$ is a positive Lipschitz constant.

\section{Time Delay Identifier Design}

In this section, auxiliary observer-like signals will be designed to facilitate the error system design and the time delay identifier will be designed subsequently. Observer-like signals, denoted by $\hat{x}_{i}(t) \in \mathbb{R} i=1, \ldots, m$, are updated according to the following rule

$$
\begin{aligned}
\dot{\hat{x}}_{1}= & \hat{x}_{2}-k_{1} \tilde{x}_{1} \\
\dot{\hat{x}}_{2}= & \hat{x}_{3}-k_{2} \tilde{x}_{2} \\
& \vdots \\
\dot{\hat{x}}_{m-1}= & \hat{x}_{m}-k_{m-1} \tilde{x}_{m-1} \\
\dot{\hat{x}}_{m}= & \hat{f}-\alpha \tilde{x}_{\varepsilon}-a^{*} \operatorname{sat}(r)
\end{aligned}
$$

where $\tilde{x}_{i} \triangleq \hat{x}_{i}-x_{i} \in \mathbb{R}, i=1, \ldots, m$ are the observer errors, $k_{i} \in \mathbb{R}, i=1, \ldots,(m-1)$ are observer gains, $\left.\hat{f} \triangleq f\right|_{\tau=\hat{\tau}}$ where $\hat{\tau}(t) \in \mathbb{R}^{n}$ is the estimate of $\tau, \alpha \in \mathbb{R}$ is a positive

\footnotetext{
${ }^{1} \mathrm{~A}$ simplex in $\mathbb{R}^{n}$ is a convex polyhedron with $n+1$ vertices.
}

constant gain, $a^{*}(t) \in \mathbb{R}$ is the tuning function, $r(t), \tilde{x}_{\varepsilon}(t) \in$ $\mathbb{R}$ are auxiliary error signals defined as

$$
\begin{aligned}
\tilde{x}_{\varepsilon} & \triangleq \tilde{x}_{m}-\varepsilon \operatorname{sat}(r) \\
r & \triangleq \tilde{x}_{m} / \varepsilon
\end{aligned}
$$

where $\varepsilon \in \mathbb{R}$ is the desired precision, and $\operatorname{sat}(\cdot) \in \mathbb{R}$ is the standard saturation function defined as follows

$$
\operatorname{sat}(z)= \begin{cases}1, & z \geqslant 1 \\ z, & |z|<1 \\ -1, & z \leqslant-1\end{cases}
$$

$\forall z \in \mathbb{R}$. Following expressions can be obtained for the time derivatives of the observer errors

$$
\begin{aligned}
\dot{\tilde{x}}_{1}= & \tilde{x}_{2}-k_{1} \tilde{x}_{1} \\
\dot{\tilde{x}}_{2}= & \tilde{x}_{3}-k_{2} \tilde{x}_{2} \\
& \vdots \\
\dot{\tilde{x}}_{m-1}= & \tilde{x}_{m}-k_{m-1} \tilde{x}_{m-1} \\
\dot{\tilde{x}}_{m}= & \hat{f}-f-\alpha \tilde{x}_{\varepsilon}-a^{*} \operatorname{sat}(r)
\end{aligned}
$$

where (1) and (4) were utilized. The following update law is proposed

$$
\dot{\hat{\tau}}=\operatorname{Proj}\left\{-\Gamma \tilde{x}_{\varepsilon} \phi^{*}\right\}
$$

where the projection strategy $\operatorname{Proj}\{\cdot\} \in \mathbb{R}^{n}$ guarantees that $\hat{\tau}(t)$ always belongs to the hypercube $\Theta$ and defined as

$$
\hat{\tau}_{j}= \begin{cases}\hat{\tau}_{j}, & \text { if } \hat{\tau}_{j} \in\left[\tau_{j, \text { min }}, \tau_{j, \text { max }}\right] \\ \tau_{j, \text { min }}, & \text { if } \hat{\tau}_{j}<\tau_{j, \text { min }} \\ \tau_{j, \text { max }} & , \quad \text { if } \hat{\tau}_{j}>\tau_{j, \text { max }}\end{cases}
$$

where the subscript $\mathrm{j}$ denotes the $j^{\text {th }}$ element of the corresponding vector $\forall j=1,2, \ldots n, \tau_{j, \min }, \tau_{j, \max } \in \mathbb{R}$ are the minimum and maximum values of the $j^{\text {th }}$ component of $\tau$, respectively, $\phi^{*}(t) \in \mathbb{R}^{n}$ is the sensitivity function, and $\Gamma \in \mathbb{R}^{n \times n}$ is a positive diagonal gain matrix. The solutions for $\phi^{*}(t)$ and $a^{*}(t)$ are obtained from a min-max optimization problem of the following form [23]

$$
\begin{aligned}
a^{*} & =\min _{\phi \in \mathbb{R}^{n}} \max _{\tau \in \tau_{s}} J \\
\phi^{*} & =\arg \min _{\phi \in \mathbb{R}^{n}} \max _{\tau \in \tau_{s}} J
\end{aligned}
$$

where $J(r, f, \hat{f}, \tau, \phi) \in \mathbb{R}$ is a performance index defined as follows

$$
J \triangleq \operatorname{sat}(r)\left[\hat{f}-f-\tilde{\tau}^{T} \Gamma \phi\right]
$$

where $\tilde{\tau}(t) \in \mathbb{R}^{n}$ is the identification error defined as follows

$$
\tilde{\tau} \triangleq \hat{\tau}-\tau .
$$

The solutions for $\phi^{*}(t)$ and $a^{*}(t)$ are obtained as: when $\tilde{x}(t)<0$

$$
\begin{aligned}
a^{*} & = \begin{cases}0 & \text { if } f \text { is concave on } \Theta_{\mathrm{s}} \\
A_{1} & \text { if } f \text { is convex on } \Theta_{\mathrm{s}}\end{cases} \\
\phi^{*} & = \begin{cases}\nabla f(\hat{\tau}) & \text { if } f \text { is concave on } \Theta_{\mathrm{s}} \\
A_{2} & \text { if } f \text { is convex on } \Theta_{\mathrm{s}}\end{cases}
\end{aligned}
$$


when $\tilde{x}(t) \geqslant 0$

$$
\begin{aligned}
& a^{*}= \begin{cases}A_{1} & \text { if } f \text { is concave on } \Theta_{\mathrm{s}} \\
0 & \text { if } f \text { is convex on } \Theta_{\mathrm{s}}\end{cases} \\
& \phi^{*}= \begin{cases}A_{2} & \text { if } f \text { is concave on } \Theta_{\mathrm{s}} \\
\nabla f(\hat{\tau}) & \text { if } f \text { is convex on } \Theta_{\mathrm{s}}\end{cases}
\end{aligned}
$$

where $A(t) \in \mathbb{R}^{(n+1)}$ is given as follows

$$
A=\left[\begin{array}{ll}
A_{1} & A_{2}
\end{array}\right]^{T}=G^{-1} b
$$

where $A_{1}(t) \in \mathbb{R}, A_{2}(t) \in \mathbb{R}^{n}$ and $G(t) \in \mathbb{R}^{(n+1) \times(n+1)}$, $b(t) \in \mathbb{R}^{(n+1)}$ are obtained as follows

$$
\begin{aligned}
G= & {\left[\begin{array}{cc}
-1 & \beta\left(\Gamma\left(\hat{\tau}-\tau_{s 1}\right)\right)^{T} \\
-1 & \beta\left(\Gamma\left(\hat{\tau}-\tau_{s 2}\right)\right)^{T} \\
\vdots & \vdots \\
-1 & \beta\left(\Gamma\left(\hat{\tau}-\tau_{s(n+1)}\right)\right)^{T}
\end{array}\right] } \\
b= & {\left[\begin{array}{c}
\beta\left(\hat{f}-f_{s 1}\right) \\
\beta\left(\hat{f}-f_{s 2}\right) \\
\vdots \\
\beta\left(\hat{f}-f_{s(n+1)}\right)
\end{array}\right] }
\end{aligned}
$$

where $\beta(\cdot) \in \mathbb{R}$ is defined as follows

$$
\beta(z)= \begin{cases}1 & \text { if } z \text { is convex on } \Theta_{\mathrm{s}} \\ -1 & \text { if } z \text { is concave on } \Theta_{\mathrm{s}}\end{cases}
$$

In $(21), f_{s h} \triangleq f\left(\tau_{s h}, x\right) \forall h=1,2, \ldots, n+1$ where $\tau_{s h} \in$ $\mathbb{R}^{n}$ are the vertices of the simplex $\Theta_{s}$. In (16) and (18), $\nabla f(\hat{\tau}) \in \mathbb{R}^{n}$ is the gradient of $f(\cdot)$ defined as follows

$$
\nabla f(\hat{\tau})=\left.(\delta f / \delta \tau)\right|_{\tau=\hat{\tau}}
$$

Remark 1: The tuning error $\tilde{x}_{\varepsilon}(t)$ and the saturation function $\operatorname{sat}(r)$ assure that the estimator is continuous even if a discontinuous solution of the min-max algorithm is obtained [23].

Remark 2: The projection strategy in (10) assures the boundedness of the $\hat{\tau}(t)$; thus, $\phi^{*}(t)$ can be upper bounded as follows

$$
\left\|\phi^{*}(t)\right\| \leqslant L_{\phi} \quad \forall t \geqslant t_{0}
$$

where $L_{\phi} \in \mathbb{R}$ is a positive constant.

Theorem 1: The observer dynamics in (4) and the adaptive update law in (9) guarantee stability and global boundedness of the closed-loop system, and $\left|\tilde{x}_{\varepsilon}(t)\right| \rightarrow 0$ as $t \rightarrow \infty$.

Proof: The proof of this theorem is lengthy, and due to space constraints is not presented in this paper, and instead the reader is referred to [24]. In [24], it was proven that $\tilde{x}_{\varepsilon}(t) \in L_{2} \cap L_{\infty}$ and $\dot{\tilde{x}}_{\varepsilon}(t) \in L_{\infty}$; thus, from Barbalat's Lemma [25], $\left|\tilde{x}_{\varepsilon}(t)\right| \rightarrow 0$ as $t \rightarrow \infty$. From its definition in (5), it is easy to see that $\left|\tilde{x}_{m}(t)\right|$ is ultimately bounded in the sense that $\left|\tilde{x}_{m}(t)\right| \leq \varepsilon$ as $t \rightarrow \infty$. Linear analysis tools can then be utilized to prove that $\left|\tilde{x}_{i}(t)\right| \leq \varepsilon$ as $t \rightarrow \infty$, $i=1,2, \ldots,(m-1)$; thus, proving ultimate boundedness of the observer errors.
Theorem 2: The estimator assures that $\|\tilde{\tau}(t)\| \leqslant \sqrt{\gamma}$ as $t \rightarrow \infty$ provided the following nonlinear persistent excitation condition holds

$$
\beta\left(x\left(t_{2}\right)\right)\left(f\left(\hat{\tau}\left(t_{1}\right), x\left(t_{2}\right)\right)-f\left(\tau, x\left(t_{2}\right)\right)\right) \geqslant \varepsilon_{u}\left\|\hat{\tau}\left(t_{1}\right)-\tau\right\|
$$

where

$$
\gamma=\frac{8 \varepsilon c_{1}}{\varepsilon_{u}^{2}} \quad ; \quad c_{1}=4 L_{1} L_{2}+2 \nu L_{2} L_{\phi}+\nu L_{\phi}^{2},
$$

where $\nu$ is maximum eigenvalue of $\Gamma, t_{2} \in\left[t_{1}, t_{1}+T_{0}\right]$, $t_{1}>t_{0}$, and $T_{0}, \varepsilon_{u} \in \mathbb{R}$ are positive constants.

Proof: The proof of this theorem is lengthy, and the reader is referred to [26] for the proof or to [24] for a similar proof. It should be noted that, unlike our work, there is no gain matrix in the adaptive update rule in [24].

Remark 3: From the definition of $\gamma$ in (26), it is clear that $\gamma$ can be made smaller by choosing a smaller $\varepsilon$. It should be noted that, as the desired precision $\varepsilon \rightarrow 0$, then $\gamma \rightarrow 0$; thus, the observer errors and the time delay identification error are driven to zero.

Remark 4: This algorithm can be applied to systems subject to input delay. In this case, the system model can be described as

$$
\begin{aligned}
\dot{x}_{1} & =x_{2} \\
\vdots & \\
\dot{x}_{m-1} & =x_{m} \\
\dot{x}_{m} & =f(x, \tau, t, u)
\end{aligned}
$$

where $u(t) \in \mathbb{R}$ is the control input. In the case of $u(t)$ being exposed to time delay(s), this time delay can also be considered as a member of time delay vector $\tau$ and can be estimated along with the state delays.

\section{Tracking Controller While IDENTIFying Time DELAYS}

In this section, we design a controller for the following general nonlinear systems

$$
\begin{aligned}
\dot{x}_{1} & =x_{2} \\
\vdots & \\
\dot{x}_{m-1} & =x_{m} \\
\dot{x}_{m} & =f(x, \tau, t)+u(t)
\end{aligned}
$$

where $u(t) \in \mathbb{R}$ is the control input. The control objective is to design $u(t)$ to guarantee that $x_{1}(t)$ tracks a desired trajectory, while identifying time delays. We can achieve this objective by redefining the error signal $\tilde{x}_{1}(t)$ as follows

$$
\tilde{x}_{1} \triangleq x_{d}-x_{1}
$$

where $x_{d}(t) \in \mathbb{R}$ is a desired trajectory. Auxiliary filtered error signals, denoted by $\tilde{x}_{i}(t) \in \mathbb{R} i=2, \ldots, m$, are defined 
as follows

$$
\begin{aligned}
\tilde{x}_{2} \triangleq \dot{\tilde{x}}_{1}+k_{1} \tilde{x}_{1} \\
\tilde{x}_{3} \triangleq \dot{\tilde{x}}_{2}+k_{2} \tilde{x}_{2} \\
\vdots \\
\tilde{x}_{m} \triangleq \dot{\tilde{x}}_{m-1}+k_{m-1} \tilde{x}_{m-1}
\end{aligned}
$$

where $k_{i} \in \mathbb{R}, i=1, \ldots,(m-1)$ are control gains. To facilitate the control design the time derivative of $\tilde{x}_{m}(t)$ can be obtained as follows,

$$
\dot{\tilde{x}}_{m}=x_{d}^{(m)}-f-u+\sum_{i=1}^{m-1} k_{i} \tilde{x}_{i}^{(m-i)}
$$

where (29), $m$-th order time derivative of (30), and (31) were utilized. The control input $u(t)$ is designed as follows

$$
u=\alpha x_{\varepsilon}+\hat{f}+a^{*} \operatorname{sat}(r)+x_{d}^{(m)}+\sum_{i=1}^{m-1} k_{i} \tilde{x}_{i}^{(m-i)} .
$$

After substituting (33) into (32), we obtain the following closed-loop error system

$$
\dot{\tilde{x}}_{m}=-\alpha x_{\varepsilon}+\hat{f}-f-a^{*} \operatorname{sat}(r) .
$$

The rest of the development is considered to continue from (9).

Remark 5: It can be seen that the expression in (34) is exactly same as the one in (8), and since the rest of the development is same, the stability analysis is valid and the proofs of Theorems 1 and 2 are applicable for this case as well. Thus, the proof of Theorem 1 ensures ultimate boundedness of the output tracking error $\tilde{x}_{1}(t)$, and the proof of Theorem 2 guarantees convergence of the time delay identification algorithm.

Remark 6: The technique presented in this paper can be considered as analogous nonlinear form of linear parameter identification. In linear parameter identification, the number of unknown parameters does not depend on the number of states [27], [28], [29]. Similarly, the dimension of the time delay vector $\tau$ in this paper does not depend on the number of states.

\section{Numerical Simulation Results}

The performance of the proposed technique was evaluated by conducting numerical simulations using Matlab/Simulink. Numerical simulation section was divided into two subsections: i) time delay identification, ii) control with time delay identification.

\section{A. Time Delay Identification}

The following model was considered

$$
\begin{aligned}
& \dot{x}_{1}(t)=x_{2}(t) \\
& \dot{x}_{2}(t)=-\left(1+x_{2}(t)\right) x_{2}(t-\tau)
\end{aligned}
$$

where $\tau$ is the time delay. While conducting the simulations, the lower and upper bounds of unknown time delay $\tau$ were chosen as 0.1 and 1.1 seconds, respectively, the initial values of $x(t)$ and $\hat{x}(t)$ were set to $[0,0.1]^{T}$ and $[0.3,0.3]^{T}$, respectively, and the initial value of $\hat{\tau}(t)$ was set to its upper bound which was 1.1 seconds.

Numerical simulation performance of the time delay identification technique was investigated for two cases. The performance of the proposed technique was evaluated with and without additive noise. In noisy case, additive white Gaussian noise with a $20 \mathrm{~dB}$ SNR was injected to $f$ to demonstrate robustness against measurement noise. During the simulations, the update law in (9) was utilized with the desired precision $\varepsilon=10^{-6}$, and the gains were chosen as $\alpha=6$ and $\Gamma=0.7$, and $k_{1}$ was chosen as 56 for the noise free case and as 52 for the noisy case.

1) Case I: The time delay was considered as constant and selected as $\tau=0.4$ seconds. In Figures 1 and 2, the estimation performances are presented for noise free and noisy cases, respectively.

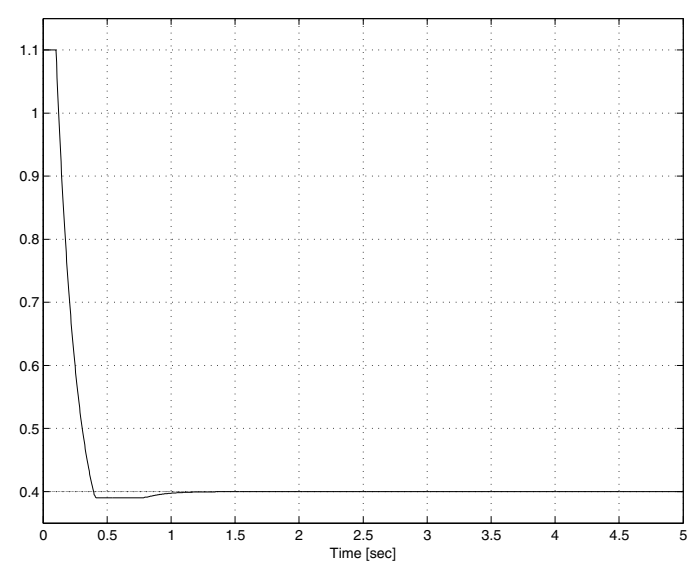

Fig. 1. The estimate of $\tau$ for case A-I without any noise

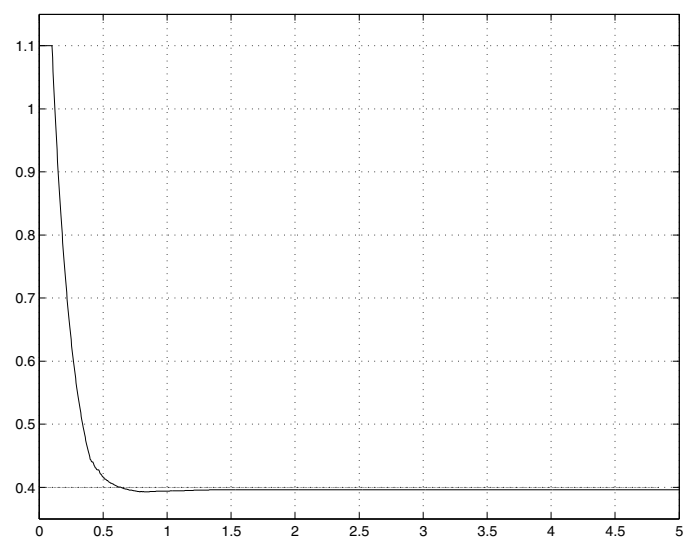

Fig. 2. The estimate of $\tau$ for case A-I with noisy measurements

2) Case II: The simulation was conducted for the following slowly time-varying time delay

$$
\tau=0.4+0.001 \sin (2 \pi 0.05 t) .
$$


In Figures 3 and 4, the estimation performances are presented for noise free and noisy cases, respectively.

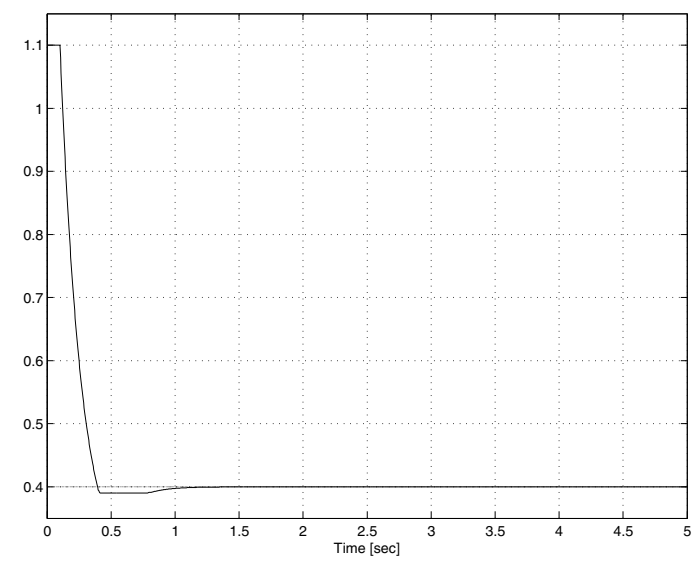

Fig. 3. The estimate of $\tau$ for case A-II without any noise

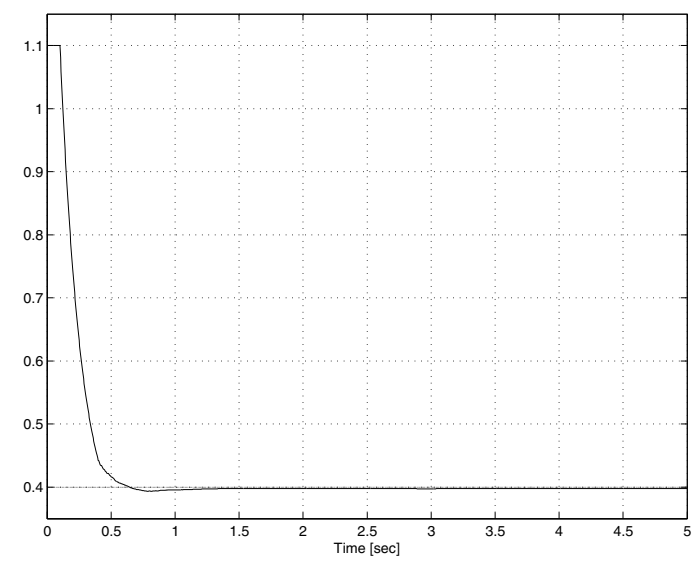

Fig. 4. The estimate of $\tau$ for case A-II with noisy measurements

\section{B. Tracking Controller while Identifying Time Delays}

The following model of chattering phenomenon during metal cutting operation was considered [30]

$$
\begin{aligned}
& \dot{x}_{1}(t)=x_{2}(t) \\
& \dot{x}_{2}(t)=m^{-1}\left(-c x_{2}-k x_{1}+k_{c} b\left(f-x_{1}+x_{1}(t-\tau)\right)+u\right)
\end{aligned}
$$

where $m, c, k, k_{c}, b, f$ are the effective mass, damping coefficient, stiffness constant, cutting stiffness, width of cut, and feed rate, respectively, and $\tau$ is the time delay. Model parameters were $m=1.16 \mathrm{~kg}, \tau=60 / \Omega, \Omega=550 \mathrm{rpm}$, $k=m \omega^{2}, c=2 m \eta \omega, \eta=0.1, \omega=83 \pi, k_{c} / k=0.5$, $b=2 \mathrm{~mm}$, and $f=0.25 \mathrm{~mm}$ per revolution [30].

The performance of the proposed technique was evaluated with and without additive noise. In noisy case, additive white Gaussian noise with a $20 \mathrm{~dB}$ SNR was injected to $f$ to demonstrate robustness against measurement noise.

During the simulations, the lower and upper bounds of unknown time delay $\tau$ were chosen as 0.05 and 0.3 seconds, respectively, the initial values of both $x(t)$ and $\hat{x}(t)$ were set to $[2,1]^{T}$, and the initial value of $\hat{\tau}(t)$ was set to its upper bound which was 0.3 seconds. The update law in (9) was utilized with the desired precision $\varepsilon=10^{-6}$, and the gains $\alpha, \Gamma$, and $k_{1}$ were chosen as $150,550,180$ for the noise free case and as 310, 105 and 90 for the noisy case, respectively. In Figures 5 and 7, and 6 and 8 estimation performances, tracking errors are presented for noise free and noisy cases, respectively.

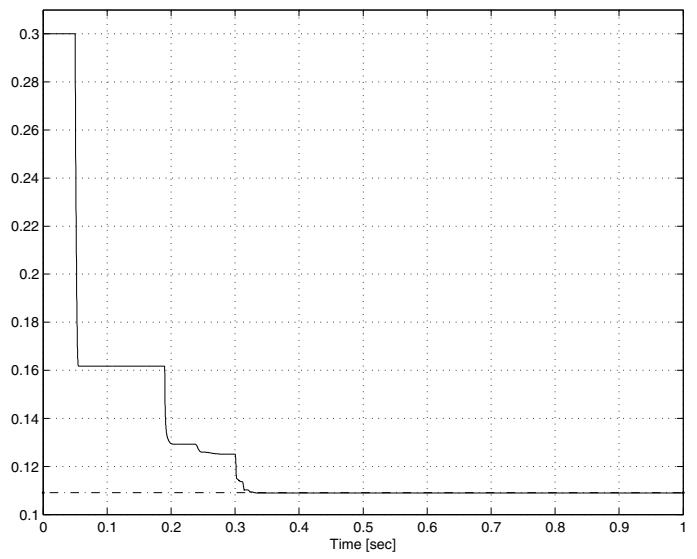

Fig. 5. The estimate of $\tau$ for noise free case

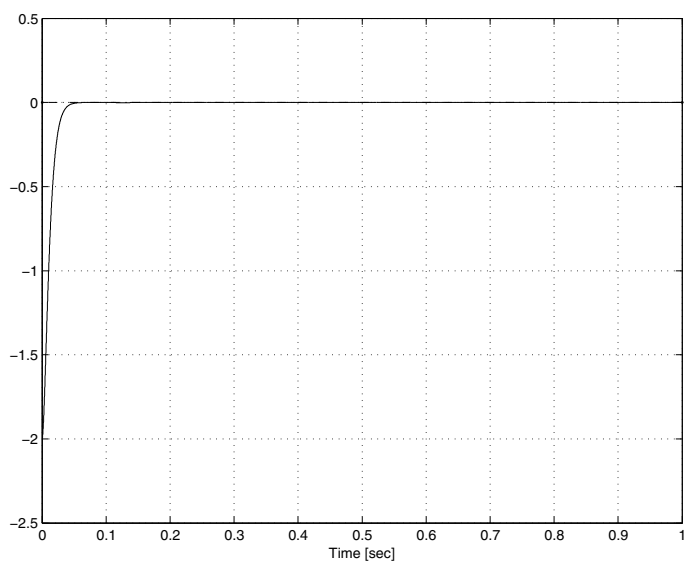

Fig. 6. The tracking error for noise free case

\section{CONCLUSION}

In this work, a novel time delay identification algorithm was proposed for general classes of autonomous nonlinear systems subject to state delays. While designing the identification algorithm, different from most of the studies in the literature, the time delay was considered as a nonlinear parameter, and the nonlinear parameter identification method in [23] was utilized as the time delay identification method. Auxiliary observer-like signals were utilized when designing 


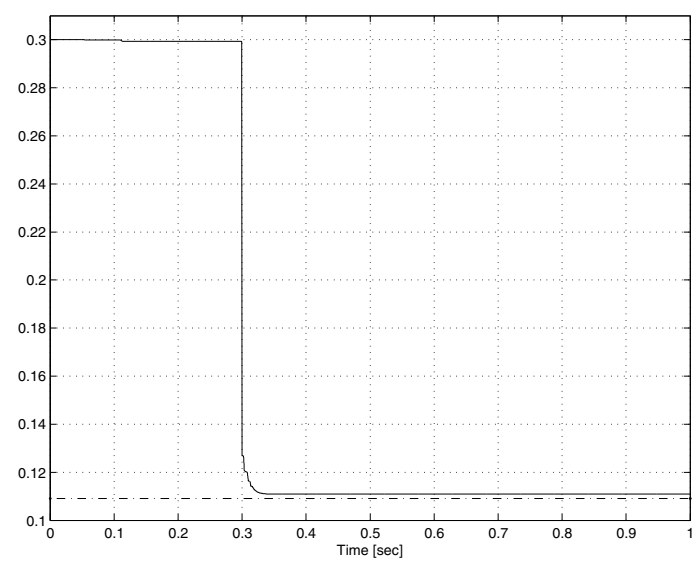

Fig. 7. The estimate of $\tau$ for noisy case

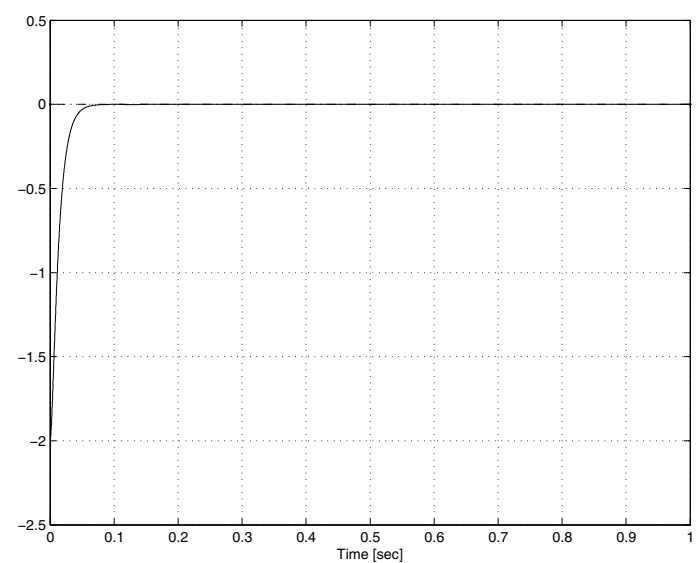

Fig. 8. The tracking error for noisy case

the time delay identification algorithm. As an extension, the time delay identification algorithm was modified to be applicable to general classes of nonlinear systems subject to state delays by designing a tracking controller. The main novelty of this design is that while the controller ensured tracking of a desired trajectory, state delays were identified online. Extensive numerical simulations were presented that demonstrated the validity of the time delay identification algorithm and the tracking controller.

\section{REFERENCES}

[1] J.-P. Richard, "Time-delay systems: an overview of some recent advances and open problems," Automatica, vol. 39, no. 10, pp. 16671694, 2003.

[2] K. Gu and S.-I. Niculescu, "Survey on recent results in the stability and control of time-delay systems," Trans. of the ASME, vol. 125, no. 2, pp. 158-165, 2003.

[3] S. Drakunov, W. Perruquetti, J.-P. Richard, and L. Belkoura, "Delay identification in time-delay systems using variable structure observers," Annual Reviews in Control, vol. 30, no. 2, pp. 143-158, 2006.

[4] S. Ahmed, B. Huang, and S. Shah, "Parameter and delay estimation of continuous-time models using a linear filter," Journal of Process Control, vol. 16, no. 4, pp. 323-331, 2006.
[5] B. Ni, D. Xiao, and S. L. Shah, "Time delay estimation for MIMO dynamical systems - with time-frequency domain analysis," Journal of Process Control, vol. 20, no. 1, pp. 83-94, 2010.

[6] S. Selvanathan and A. Tangirala, "Time-delay estimation in multivariate systems using hilbert transform relation and partial coherence functions," Chemical Engineering Science, vol. 65, no. 2, pp. 660-674, 2010.

[7] L. Belkoura and J.-P. Richard, "A distribution framework for the fast identification of linear systems with delays," in Proc. of 6th IFAC Workshop on Time Delay Systems, L'Aquila, Italy, 2006.

[8] Y. Wang, Y. Zhao, X. Hao, and P. Cheng, "Time-delay identification for linear multi-input multi-output systems," in Proc. of the 7th World Congress on Intelligent Control and Automation, Chongqing, China, 2008, pp. 4762-4766.

[9] R. Loxton, K. Teo, and V. Rehbock, "An optimization approach to state-delay identification," IEEE Tran. on Automatic Control, vol. 55, no. 9, pp. 2113-2119, 2010.

[10] S. Bjorklund and L. Ljung, "An improved phase method for time-delay estimation," Automatica, vol. 45, no. 10, pp. 2467-2470, 2009.

[11] Y. Tang, M. Cui, L. Li, H. Peng, and X. Guan, "Parameter identification of time-delay chaotic system using chaotic ant swarm," Chaos, Solitons and Fractals, vol. 41, no. 4, pp. 2097-2102, 2009.

[12] Y. Tang and X. Guan, "Parameter estimation of chaotic system with time-delay: A differential evolution approach," Chaos, Solitons \& Fractals, vol. 42, no. 5, pp. 3132-3139, 2009.

[13] A. H. Tan and C. L. Cham, "Continuous-time model identification of a cooling system with variable delay," IET Control Theory and Applications, vol. 5, no. 7, pp. 913-922, May 2011.

[14] A. Bayrak and E. Tatlicioglu, "A novel online adaptive time delay identification technique," in Proc. of Conf. on Decision and Control, Orlando, FL, USA, 2011.

[15] Q.-C. Zhong, Robust control of time-delay systems. Germany: Springer, 2001.

[16] G. M. Schoen, Stability and Stabilization of Time-Delay Systems. Zurich, Switzerland: Swiss Federal Institute of Technology, 1995.

[17] M. Krstic, Delay Compensation for Nonlinear, Adaptive, and PDE Systems. Boston, MA, USA: Birkhauser, 2009.

[18] S.-I. Niculescu, Delay effects on stability: A robust control approach. Germany: Springer, 2001.

[19] F. Mazenc, M. Malisoff, and Z. Lin, "Further results on input-to-state stability for nonlinear systems with delayed feedbacks," Automatica, vol. 44 , no. 9 , pp. $2415-2421,2008$.

[20] C. Peng, D. Yue, and J. Sun, "The study of smith prediction controller in ncs based on time-delay identification," Control, Automation, Robotics and Vision Conference, pp. 1644-1648, 2004.

[21] T. Zhang and Y.-C. Li, "A fuzzy smith control of time-varying delay systems based on time delay identification," in Proc. of International Conference on Machine Learning and Cybernetics, Changchun, China, July 2003.

[22] T. Zhang and Y. Li, "A control scheme for bilateral teleoperation systems based on time-varying communication delay identification," in Proc. of Systems and Control in Aerospace and Astronautics, Harbin, China, 2006, pp. 273-278.

[23] A. M. Annaswamy, F. P. Skantze, and A.-P. Loh, "Adaptive control of continuous time systems with convex/concave parametrization," Automatica, vol. 34, no. 1, pp. 33-49, 1998.

[24] N. Nath, E. Tatlicioglu, and D. M. Dawson, "Range identification for nonlinear parameterizable paracatadioptric systems," Automatica, vol. 46, no. 7, pp. 1129-1140, 2010.

[25] H. K. Khalil, Nonlinear Systems. NJ, USA: Prentice Hall, 2002.

[26] A. Bayrak and E. Tatlicioglu, "Adaptive identification of constant or slowly varying time delays for general classes of nonlinear systems while guaranteeing output tracking," Izmir Institute of Technology, Tech. Rep., 2011. [Online]. Available: http://web.iyte. edu.tr $\backslash \sim$ envertatlicioglu/TR/IYTE-NC-06-11.pdf

[27] P. Ioannou and J. Sun, Robust Adaptive Control. Englewood Cliffs, NJ, USA: Prentice-Hall, 1996.

[28] P. Ioannou and B. Fidan, Adaptive Control Tutorial. Philadelphia PA, USA: Cambridge University Press, 2006.

[29] S. Sastry and M. Bodson, Adaptive Control: Stability, Convergence and Robustness. Englewood Cliffs, NJ, USA: Prentice-Hall, 1989.

[30] N. Sharma, S. Bhasin, W. Qiang, and W. Dixon, "RISE-based adaptive control of a control affine uncertain nonlinear system with unknown state delays," IEEE Tran. on Automatic Control, vol. 57, no. 1, pp. 255-259, 2012. 